\title{
Enhanced superchannel transmission using phase conjugation
}

A. D. Ellis ${ }^{\left(1,{ }^{*}\right)}$ I. D. Phillips ${ }^{(1)}$, M. Tan ${ }^{(1)}$, M. F. C. Stephens ${ }^{(1)}$, M. E. McCarthy ${ }^{(1)}$, M. A. Z. Al Kahteeb ${ }^{(1)}$, M.A. Iqbal ${ }^{(1)}$, A. Perentos ${ }^{(1)}$, S. Fabbri ${ }^{(1)}$, V. Gordienko ${ }^{(1)}$, D. Lavery ${ }^{(2)}$, G. Liga'(2) G. Saavedra. M. ${ }^{(2)}$,' R. Maher ${ }^{(2)}$, S. Sygletos ${ }^{(1)}$, P. Harper ${ }^{(1)}$, N. J. Doran ${ }^{(1)}$, P. Bayvel ${ }^{(2)}$, S. K. Turitsyn ${ }^{(1)}$

(1) Aston Institute of Photonic Technologies, Aston University, Birmingham, B4 7ET, England

(2) Optical Networks Group, University College London, Torrington Place, London, WC1E 7JE, UK, * andrew.ellis@aston.ac.uk

Abstract We demonstrate polarisation insensitive dual-band optical phase conjugation for multiple 400Gbit/s optical superchannels using a Raman amplified transmission link with a realistic span length of $75 \mathrm{~km}$. The resultant increase in transmission distance is confirmed analytically.

\section{Introduction}

In order to maximise the capacity of single mode fibre based optical communication systems it is necessary to simultaneously minimise amplified spontaneous emission using distributed amplification $^{1}$, maximise spectral efficiency using superchannels ${ }^{2}$ and fully compensate for all deterministic nonlinear impairments ${ }^{3}$. Whilst Raman amplification and superchannels are becoming established, the optimum means to compensate nonlinearity is the focus of much research. Candidates include wide-bandwidth digital back propagation ${ }^{4}$, phase conjugate coding $^{5}$ and optical phase conjugation (OPC) ${ }^{6-8}$. OPC uniquely offers disruption of parametric noise amplification ${ }^{3}$ thus overcomes the nonlinear Shannon limit $^{7}$ and $60 \%$ reach enhancement has been shown for a $1 \mathrm{Tbit} / \mathrm{s}$ signal $^{8}$. However, to maximise capacity, it is important for OPC systems to operate on two signal bands simultaneously.

In this paper, we experimentally demonstrate for the first time the combination of polarisation multiplexed 16QAM optical superchannels, lossless Raman amplification and dual-band optical phase conjugation, resulting in a reach in excess of $2,700 \mathrm{~km}$ for multiple $400 \mathrm{Gbit} / \mathrm{s}$ Nyquist superchannels with a record total bit rate of $2.4 \mathrm{Tbit} / \mathrm{s}$. This is the first report where both wavebands are simultaneously conjugated, and the first report of OPC of multiple optical superchannels.

\section{Experimental configuration}

Figure 1 shows the experimental configuration including the 400Gbit/s Nyquist WDM superchannel transmitter; the dual band OPC, the Raman laser based transmission link and the recirculating loop configuration. For WDM transmission, a combination of DFB, external cavity and fibre lasers formed two groups of up to 5 lasers each centred at 193 and $194.6 \mathrm{THz}$ with $100 \mathrm{GHz}$ spacings. For each measurement the DFB for that superchannel was replaced with an external cavity laser. The 10 independent lasers were multiplexed together and subsequently modulated with Nyquist shaped 10Gbaud (2 samples per symbol) 16QAM in-phase (I) and quadrature (Q) electrical signals (with $2^{15}$ bit sequences) using an IQ modulator. The digital Nyquist filter had a roll off factor of 0.01 at the output of the arbitrary waveform generator. The resulting optical Nyquist signals were amplified and passed into a Mach Zehnder modulator (MZM) driven at $20.2 \mathrm{GHz}$ and biased to provide a 3 line comb. These $20.2 \mathrm{GHz}$ spaced signals were split and one copy was frequency shifted by $10.1 \mathrm{GHz}$ using a single side band modulation scheme with over $30 \mathrm{~dB}$ extinction ratio. The other copy was delayed by 5 symbols before recombining to form a superchannel with a spectral width of approximately $60 \mathrm{GHz}$. After amplification, the signals were polarisation multiplexed with a 400ps relative delay. The gross data rate for a superchannel was $480 \mathrm{Gbit} / \mathrm{s}$, a net data rate of

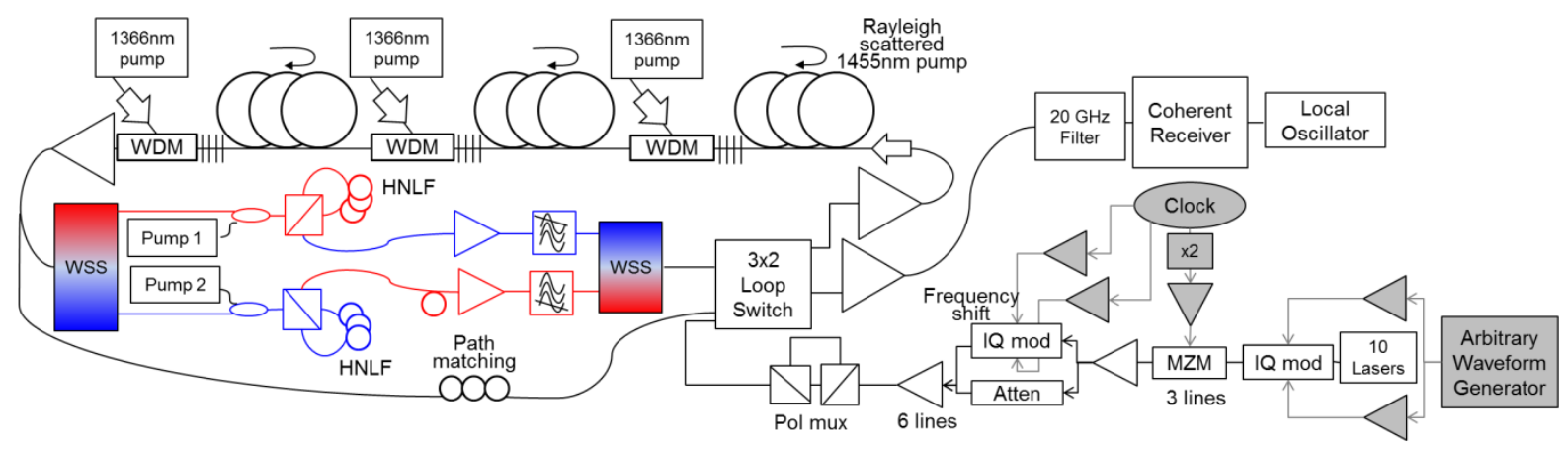

Fig. 1: Schematic diagrams of transmitter, second order Raman amplified loop and dual band OPC. 
$400 \mathrm{Gbit} / \mathrm{s}$ after $20 \%$ overhead removal for FEC. The signals were launched into a multi-path recirculating loop via an erbium doped fibre amplifier and passed through three Raman laser based spans. In each of the $75 \mathrm{~km}$ Sterlite $\mathrm{OH}$ LITE $^{\text {TM }}(\mathrm{E})$ fibre spans, the $1452 / 1455 \mathrm{~nm}$ pump (the spread of wavelengths enhancing the gain flatness) was generated by lasing in a cavity formed by a fibre grating at the fibre output, and distributed Rayleigh scattering throughout the link, pumped by a $1366 \mathrm{~nm}$ fibre laser with $1.1 \mathrm{~W}$ launched power in each span. This gave a near symmetric power profile to maximise the efficiency of OPC-based nonlinearity compensation $^{9}$ as illustrated in Fig 2, which shows an OTDR measurement of the second span plotted both forwards and backwards. The same data is duplicated for the first and second span to illustrate the impact of coupler losses. A $6 \mathrm{~dB}$ power excursion and power asymmetry of only $18 \%$ is observed over the $3 \times 75 \mathrm{~km}$ link. Since each individual span has asymmetry of less than $8 \%$ the majority of the asymmetry is attributed to the finite loss the pump couplers and fibre gratings ( $0.5 \mathrm{~dB}$ per span).

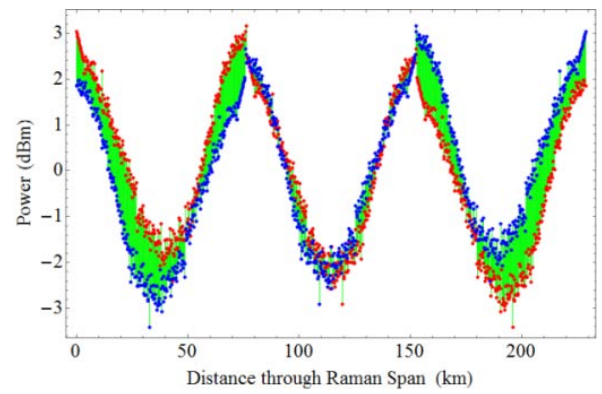

Fig. 2: Signal power evolution through one span (red) and with direction reversed (blue). Trace repeated every $75 \mathrm{~km}$ with $0.5 \mathrm{~dB}$ coupler loss. Asymmetry highlighted in green.

The signals propagated through this link a number of times (which included a gain flattening filter and an amplifier to overcome loop specific losses), before entering the dual OPC. Here the two groups of $5 \times 400 \mathrm{Gbit} / \mathrm{s}$ superchannels were amplified, split into two parallel paths using a wavelength selective switch (WSS), giving $\sim 12 \mathrm{dBm}$ total power per band, and conjugated in two nearly identical single pump polarisation-diverse OPCs ${ }^{10}$. The OPCs used independent pump lasers and aluminium doped highly-nonlinear fibres with lengths, fibre loss and nonlinear coefficients of $100 \mathrm{~m}, 6.3 \mathrm{~dB} / \mathrm{km}$ and $6.9 /(\mathrm{W} . \mathrm{km})$ respectively. At the output of each OPC, the residual pumps were blocked using thin-film notch filters and the conjugated bands gain-equalised and combined using a second WSS. Unlike previous experiments $^{6-8}$ this ensured that all channels were launched simultaneously, and that intraband nonlinearity compensation was performed on both bands simultaneously. After propagating through the Raman spans for an identical number of recirculations, a portion of the superchannel under test was filtered using a $20 \mathrm{GHz}$ optical filter, detected using a conventional coherent receiver with $26 \mathrm{GHz}$ analogue bandwidth and processed using conventional digital signal processing code optimised for Nyquist signals ${ }^{11}$.

\section{Results}

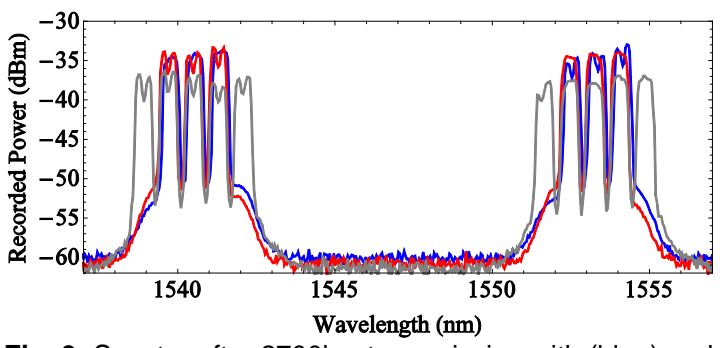

Fig. 3: Spectra after 2700km transmission with (blue) and $2025 \mathrm{~km}$ without (red) OPC for-6x400Gbit/s superchannels. Also shows spectra with OPC for 10 superchannels (grey).

Typical transmission spectra for signals propagated with $(2700 \mathrm{~km})$ and without $(2025 \mathrm{~km})$ OPC, are shown in Fig. 3. The high quality of the super channel generation, and the amplitude of the unwanted sidebands (more than $20 \mathrm{~dB}$ suppressed) can be seen clearly, along with the gain flatness of the Raman amplified loop. Slight dips for the shorter wavelength superchannels correspond to bias point errors.

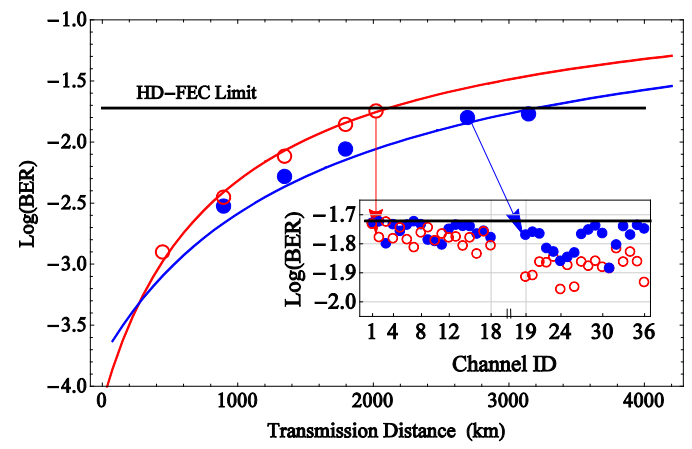

Fig. 4: Measured (dots) and calculated (lines) performance of central channel with (blue) and without (red) OPC for six superchannels versus transmission distance. Inset: BER of all sub-channels at maximum reach.

The transmission performance of the system when propagating 6 superchannels is shown in Fig. 4, which includes plots of BER as a function of transmission distance for the central subchannel of the $1553 \mathrm{~nm}$ group, and the BER performance at the maximum transmission distance with and without OPC for all sub channels. Launch powers were optimised at $1350 \mathrm{~km}$ to $4 \mathrm{dBm} / 1 \mathrm{dBm}$ with/without OPC. The reach enhancement was approximately $55 \%$.

Curve fits are based on analytical estimates ${ }^{9}$ taking into account inter- and intra-signal 
nonlinearities, parametric noise amplification ${ }^{3}$, and the transmitter SNR (which decreased as the number of superchannels increased). The nonlinear compensation only took into account one band of signals (since the second band was neither time nor polarisation aligned in the dual path OPC), reduced by the impact of polarisation mode dispersion (PMD) $)^{12}$ and power asymmetry $^{7}$. Signal to noise ratios were converted to BER assuming Gaussian noise distributions and Gray mapping. The curve fits correspond to a Raman noise power spectral density of $10^{-20} \mathrm{~W} /(\mathrm{km} . \mathrm{Hz})$ and $0.1 \mathrm{ps} / \sqrt{\mathrm{km}} \mathrm{PMD}$. The effective nonlinear coefficient integrated over the signal spectrum $\left(\eta\right.$ in $\left.^{9}\right)$ and the transmitter SNR varied with the number of superchannels. For one (five) superchannel(s) these coefficients were 20 (16.5) dB and 0.20 (0.22) $\mathrm{THz}^{2} /\left(\mathrm{W}^{2} . \mathrm{km}\right)$ respectively. All other parameters were measured from the experimental set up. We observe a good fit between experimental and theoretical results.

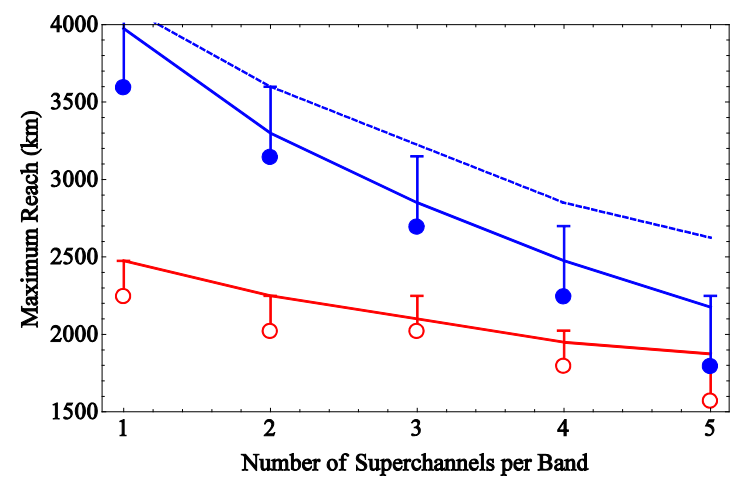

Fig. 5: Measured (dots) and calculated (solid lines, dashed line without PMD) reach with (blue) and without (red) OPC.

Error bars reflect the length of each loop circulation.

The dependence of reach enhancement on the system configuration is illustrated in Fig. 5, where we plot the reach for a pre FEC BER of $\leq 1.9 \times 10^{-2}$ with and without dual band OPC (central sub-channel in each superchannel of one band measured). Again, excellent agreement was achieved between theory and experiment (limited to a step size of $450 \mathrm{~km}$ with and $225 \mathrm{~km}$ without OPC), as shown by the blue curves (theory) and the dots (experiment). Theoretically the reach decreases with increasing number of superchannel since the nonlinear threshold decreases and depends on the signal to noise ratio without $\mathrm{OPC}^{9}$. For an ideal OPC, the maximum reach should exceed $7,500 \mathrm{~km}$, but if only the nonlinearity of one band is compensated; this reduces to $5,000 \mathrm{~km}$. The observed power fluctuations between spans $( \pm 0.5 \mathrm{~dB}$ in this case) further degrades the performance to less than $4,000 \mathrm{~km}$ (Fig 5 . dashed line). PMD reduces the reach by a further $300 \mathrm{~km}$ (Fig 5. solid line).

\section{Conclusions}

In this paper we have demonstrated, for the first time, dual channel optical phase conjugation of an optical superchannel using $75 \mathrm{~km}$ spans of standard single mode fibre. We are able to substantially eliminate inter-channel nonlinear penalties. This nonlinearity compensation allows a $\sim 50 \%$ increase in reach for 6 simultaneously transmitted 400Gbit/s 16QAM superchannels, a record total bit rate $2.4 \mathrm{Tbit} / \mathrm{s}$ using a single dualband OPC. 8 and 10 superchannels (3.2 and 4Tbit/s) transmission also appears feasible.

\section{Acknowledgements}

This work was partly funded by the ECs 7th Framework Programme grant 318415 (FOX-C), the EPSRC projects EP/J017582/1, EP/L000091/1 \& EP/J009709/1 (UNLOC, PEACE and FOPA) and The Royal Society (WM120035-TEST). The authors thank Sterlite Technologies and Finisar for their support.

\section{References}

[1] A. Carena et al.,"On the Optimisation of Hybrid Raman/Erbium-Doped Fiber Amplifiers", Photon. Technol. Lett., 13, 11, (2001).

[2] D.Qian et al," 101.7-Tb/s (370×294-Gb/s) PDM-128QAMOFDM Transmission over $3 \times 55-k m$ SSMF using Pilotbased Phase Noise Mitigation", OFC2011, PDPB5 (2011)

[3] D. Rafique et al.," Impact of signal-ASE four-wave mixing on the effectiveness of digital back-propagation in 112 Gb/s PM-QPSK systems", Opt. Express, 19, 4, (2011)

[4] G. Liga, et al, "On the performance of multichannel digital backpropagation in high-capacity long-haul optical transmission", Opt. Express 22, 30053 (2014)

[5] T. Yoshida, "Spectrally-efficient dual phase-conjugate twin waves with orthogonally multiplexed quadrature pulse-shaped signals", OFC 2014, paper M3C.6 (2014)

[6] I.Phillips et al.," Exceeding the nonlinear-Shannon limit using Raman laser based amplification and optical phase conjugation", OFC2014, paper M3C1 (2014).

[7] K.Solis-Trapala, et al., "Transmission Optimized Impairment Mitigation by 12 Stage Phase Conjugation of WDM 24x48 Gb/s DP-QPSK Signals",OFC 2015 ,Th3C.2.

[8] I. Sackey et al., "Kerr Nonlinearity Mitigation: Mid-Link Spectral Inversion versus Digital Backpropagation in $5 \times 28$ GBd PDM 16-QAM Signal Transmission", JLT, 33, 9, (2015).

[9] A.D.Ellis et al., "Capacity limits in nonlinear fiber transmission", ACP2014,paper AW4F1 (2014).

[10] M.F.C.Stephens et al., "1.14Tb/s DP-QPSK WDM polarization-diverse optical phase conjugation", Opt.Express 22,10 (2014).

[11] R.Maher et al., "Spectrally Shaped DP-16QAM SuperChannel Transmission with Multi-Channel Digital Back Propagation", Scientific Reports ,5, 8214, (2015).

[12] A.D.Ellis et.al.," The Impact of Phase Conjugation on the Nonlinear-Shannon Limit", IEEE Sum. Topicals (2015). 